\title{
Informal caregiving and markers of adiposity in the UK Household Longitudinal Study
}

\author{
Rebecca E. Lacey*, Anne McMunn, Elizabeth Webb \\ Research Department of Epidemiology and Public Health, University College London, London, United \\ Kingdom \\ * rebecca.lacey@ucl.ac.uk
}

\section{Abstract}

\section{Objectives}

The aim was to investigate associations between caregiving and adiposity using a representative UK longitudinal study. We also investigated whether associations differed by age, gender and caregiving characteristics.

\section{open access}

Citation: Lacey RE, McMunn A, Webb E (2018) Informal caregiving and markers of adiposity in the UK Household Longitudinal Study. PLOS ONE 13 (7): e0200777. https://doi.org/10.1371/journal. pone.0200777

Editor: Clemens Fürnsinn, Medical University of Vienna, AUSTRIA

Received: February 7, 2018

Accepted: June 6, 2018

Published: July 19, 2018

Copyright: @ 2018 Lacey et al. This is an open access article distributed under the terms of the Creative Commons Attribution License, which permits unrestricted use, distribution, and reproduction in any medium, provided the original author and source are credited.

Data Availability Statement: All UK Household Longitudinal Study datafiles are held at the UK Data Service (https://discover.ukdataservice.ac.uk/ series/?sn=2000053).

Funding: This work was funded by the UK's Economic and Social Research Council (grant number: ES/N012054/1, PI: Elizabeth Webb). The Economic and Social Research Council funded the UK Household Longitudinal Study. The funders had no role in the study design of this specific study, analysis, decision to publish, or preparation of the manuscript.

\section{Methods}

Data on 9,421 participants aged 16+ from three waves (2009-2012) of the UK Household Longitudinal Study were used. Body mass index, waist circumference and percentage body fat were assessed. Caregiving and caregiving characteristics (hours per week, number of people cared for, co-resident caregiving and combining working and caregiving) was available from the prior wave. Gender-stratified associations between caregiving/caregiving characteristics with adiposity were tested. Covariates included caregiver's health, socioeconomic position, parenthood and partnerships.

\section{Results}

Caregiving was associated with higher adiposity for women but not men. Younger women caregivers had particularly higher levels of adiposity. Men combining part-time paid work with caregiving had higher levels of adiposity than men working full-time and not caregiving. Women aged 16-44 or 65+ had particularly high levels of adiposity when combining fulltime work and caregiving, compared to full-time work alone.

\section{Conclusion}

The health of caregivers should be a public health priority, particularly for younger women and those combining paid work with caregiving responsibilities. 
Competing interests: The authors have declared that no competing interests exist.

\section{Introduction}

There are approximately seven million informal caregivers in the United Kingdom (UK) and an estimated $20 \%$ of the adult population in the United States providing informal care, $60 \%$ of whom are women in both countries [1,2]. Informal caregiving has large economic importance; the value of care in the United States in 2009 was estimated at $\$ 450$ billion, much larger than the $\$ 361$ billion spent on Medicare that year [3]. Similarly carers have been estimated to save the UK economy $£ 132$ billion per year [1]. Informal caregiving is increasing over time in response to rising life expectancy [4] and adult social care funding cuts [5].

Meta-analyses conclude that, on average, caregiving is associated with poorer psychological and physical health [6-8]. However the caregiving research has largely utilised cross-sectional studies, samples of older adults or focussed on caregivers to specific patient groups, such as dementia or cancer sufferers [8]. Caregiving is linked to poorer health through the uptake of risky health behaviours, physical strain and fatigue, reduction in social support, loss of selfidentity, change in the nature and reciprocity of the caregiver-care recipient relationship (particularly in the case of caring for someone with dementia), caregiver burden, psychological distress, conflict between caregiving and work responsibilities, and financial burden, for example due to reduced hours of paid work [9-14]. These mechanisms may accompany, and feed into, a physiological stress mechanism through hypothalamic-pituitary-adrenal axis dysregulation. Previous research has shown that caregivers have higher salivary cortisol compared to noncaregivers [15]. One manifestation of this stress mechanism is increased fat deposition [16]. Vitaliano and colleagues [12] investigated the association between caregiving for a spouse with Alzheimer's Disease and body mass index (BMI) in the United States. Caregivers experienced an increase in BMI, partly explained by calorific intake and perceived control. Also Hajek and Konig [17] explored the association between informal caregiving and change in BMI over five waves of the German Aging Survey (adults aged 40 years and over), finding that BMI increased when men initiated caregiving but not women. The association between caregiving and adiposity is yet to be examined in a broader population of the UK population aged 16+ and not restricted to caregivers providing care for people with specific health conditions, such as dementia.

Associations between informal caregiving and health depend upon several factors. Firstly, the caregiving intensity shows graded relationships with health. For example, UK data showed that caregivers providing fewer than 10 hours per week of care did not have worse health than non-caregivers [18,19], and in one study was associated with lower mortality [20]. Combining caregiving with work presents additional challenges and this combination is becoming increasingly common with extended working lives [21]. Unlike other social roles, caregiving is relatively inflexible, often requiring tasks to be completed at specific times [22]. This social role combination is likely to have health implications. For instance, caregivers who were employed had poorer self-rated health [23]. This has yet to be investigated in relation to an objective measure of health, such as adiposity. Gender is also important. Women are more likely to be caregivers from early adulthood to early old age [24], but men are more likely to be caregivers in older age [25]. Women caregivers are more likely to report poorer health than male caregivers [6], often because they have more intense caregiving responsibilities [26] and consequently report higher caregiver burden and stress [27]. Women caregivers are also more likely to reduce their working hours or leave paid work for caregiving activities, and this is further linked to higher caregiving intensity [21]. Finally, age is important but previous findings have been inconsistent in this respect. There is a suggestion that older caregivers have poorer health due to less social interaction outside of the caregiver-care recipient relationship, less use of formal support systems and reduced financial, psychological and physical resources [8]. One 
meta-analysis [8] found that older caregivers had worse physical health than younger caregivers. However the authors also found that younger caregivers reported more depression and caregiver burden, possibly because of the competing demands of caregiving with work and family. Further research is required which examines the importance of age.

The aim of the present study was to assess whether caregiving was associated with higher adiposity in a nationally representative, longitudinal sample of people aged $16+$. We also investigated whether caregiving characteristics, such as caregiving hours, the number of people cared for, whether caregiving was combined with paid work, exhibited graded associations with adiposity, and whether associations between caregiving and adiposity were modified by age.

\section{Methods}

\section{Data}

This study used data from the UK Household Longitudinal Study (UKHLS), a large nationally representative sample of 40,000 UK households initiated in 2009 [28]. The UKHLS has a panel design with a stratified, clustered, equal probability sample. The study incorporates participants of the British Household Panel Study initiated in 1991. All adults aged 16+ in each household are surveyed every year. A health assessment was performed in 2010-2012 (waves 2 and 3) for a sub-sample of participants who resided in Great Britain (England, Scotland and Wales) and who had completed a full interview in English at the previous wave $(n=20,644$, 15,591 of whom were UKHLS participants (wave 2) and 5,053 were originally British Household Panel Study participants (wave 3)).[29] This assessment included measures of adiposity (described further below) and so this study used data from waves 1-3 (2008-2012).

\section{Ethics statement}

Written informed consent was obtained from all study participants for all waves. Ethical approval was obtained from the University of Essex ethics Committee.

\section{Measures}

Caregiving and caregiving characteristics. All participants were asked at each survey whether they looked after anyone who was sick, disabled or elderly either within or outside of the household. This information was used to indicate whether participants were caregivers or non-caregivers at waves 1 and 2. Caregivers were subsequently asked to report the number of hours per week spent caregiving. Caregiving hours were categorised as not caregiving, 1-9, $10-19$, or $20+$ hours per week. These categories were based on existing literature and the available banded caregiving hours available. Additional caregiving variables included the number of people being cared for (categorised as not caregiving, one person or two or more people), whether the caregiver was caregiving for someone inside the household (co-resident caregiving) or just outside of the household, and the combination of caregiving with paid work (not working or caregiving, caregiving but not working, caregiving with part-time work $(\leq 30$ hours/week), caregiving with full-time work ( $>30$ hours/week), working part-time but not caregiving and working full-time but not caregiving).

Adiposity. Three adiposity measures were available from the nurse visit-BMI, waist circumference and percentage body fat. BMI was calculated using measured height and weight as weight $(\mathrm{kg}) /$ height(metres) $)^{2}$. Waist circumference was measured three times by a study nurse and the mean across all three was used. Percentage body fat was measured using bioelectrical impedance using a digital floor scale on a hard floor surface. 19,872 participants had a valid 
BMI measure, 20,294 had a valid waist measurement and 18,808 had a valid body fat measurement.

Covariates. Covariates were gender, banded age (16-44, 45-64, or 65+ years), presence of a longstanding illness or disability, educational attainment (the highest qualification attained: no qualifications, General Certificate of Secondary Education/Ordinary-level qualifications or equivalent, Advanced-level qualification or equivalent, or degree or higher qualification), social class (National Statistics Socio-Economic Classification (NS-SEC) of current or last job: management and professional occupation, intermediate occupation or routine occupation/ never worked), partnership status (single, married/civil partnership and living with a partner, separated or divorced, widowed, or cohabiting), number of dependent children aged 18 or under, net equivalised household income per month (quintiles), and smoking status (never-, ex- or current smoker). Household income was chosen over individual income, as it is more likely to reflect available household resources for the purchasing of formal care. By equivalising household income we have taken into account household composition. All variables, with the exception of smoking status which was only available at wave $2 / 3$ and adiposity, were used at the survey prior to the health assessment (wave 2 for the UKHLS sample component and wave 3 for the British Household Panel Study sample component, referred to hereafter as ' $t 1$ ').

Missing data. Participants with complete data on caregiving, covariates and adiposity were included in the analyses. Table 1 shows the number of missing values on each variable of interest and how the complete case sample differed from those with observed data. This exploration shows that our sample was less likely to be caregiving and were more socio-

Table 1. Characteristics of the study sample.

\begin{tabular}{|c|c|c|c|c|c|c|c|c|}
\hline & \multicolumn{2}{|l|}{ Men } & \multicolumn{2}{|c|}{ Women } & \multirow[b]{2}{*}{$\begin{array}{l}P \text { gender } \\
\text { difference }\end{array}$} & \multirow[b]{2}{*}{$\begin{array}{c}\text { Distribution of excluded } \\
\text { sample } \\
\text { Mean } / \%\end{array}$} & \multirow[b]{2}{*}{$\begin{array}{l}\text { Number } \\
\text { missing }\end{array}$} & \multirow[b]{2}{*}{$\begin{array}{l}P \text { compared to whole } \\
\text { sample }\end{array}$} \\
\hline & $\mathbf{N}$ & $\%$ & $\mathbf{N}$ & $\%$ & & & & \\
\hline \multicolumn{9}{|l|}{ Outcomes } \\
\hline BMI, mean (SD) & 28.1 & 4.9 & 27.8 & 6.0 & 0.005 & 27.6 & 611 & 0.004 \\
\hline Waist, mean (SD) & 99.2 & 13.3 & 88.6 & 14.1 & $<0.001$ & 92.9 & 189 & 0.279 \\
\hline \% body fat, mean (SD) & 23.5 & 9.3 & 35.9 & 8.2 & $<0.001$ & 29.7 & 1675 & $<0.001$ \\
\hline \multicolumn{9}{|l|}{ Caregiving } \\
\hline \multicolumn{9}{|l|}{ Caregiving } \\
\hline No & 3502 & 89.9 & 4637 & 85.7 & $<0.001$ & 69.8 & 4650 & $<0.001$ \\
\hline Yes & 432 & 10.1 & 850 & 14.3 & & 30.2 & & \\
\hline \multicolumn{9}{|l|}{ Caregiving hours } \\
\hline Not caregiving & 3502 & 89.9 & 4637 & 85.7 & $<0.001$ & 65.7 & 4217 & $<0.001$ \\
\hline 1-9 hours/week & 345 & 8.1 & 585 & 9.1 & & 16.8 & & \\
\hline 10-19 hours/week & 43 & 1.1 & 139 & 1.8 & & 5.3 & & \\
\hline $20+$ hours/week & 44 & 0.9 & 126 & 1.6 & & 12.2 & & \\
\hline \multicolumn{9}{|l|}{ Number of people cared for } \\
\hline Not caregiving & 3502 & 89.9 & 4637 & 85.7 & $<0.001$ & 79.3 & 5249 & $<0.001$ \\
\hline 1 person & 297 & 6.8 & 585 & 9.8 & & 14.6 & & \\
\hline $2+$ people & 135 & 3.3 & 265 & 4.5 & & 6.2 & & \\
\hline \multicolumn{9}{|l|}{ Combining work with caregiving } \\
\hline Not working or caregiving & 1337 & 30.8 & 1911 & 34.6 & $<0.001$ & 41.4 & 6015 & $<0.001$ \\
\hline Caregiving but not working & 185 & 3.9 & 349 & 5.9 & & 21.8 & & \\
\hline Caregiving with PT work & 32 & 0.8 & 274 & 4.5 & & 4.3 & & \\
\hline
\end{tabular}


Table 1. (Continued)

\begin{tabular}{|c|c|c|c|c|c|c|c|c|}
\hline & \multicolumn{2}{|l|}{ Men } & \multicolumn{2}{|c|}{ Women } & \multirow[b]{2}{*}{$\begin{array}{l}P \text { gender } \\
\text { difference }\end{array}$} & \multirow[b]{2}{*}{$\begin{array}{c}\text { Distribution of excluded } \\
\text { sample } \\
\text { Mean } / \%\end{array}$} & \multirow[b]{2}{*}{$\begin{array}{l}\text { Number } \\
\text { missing }\end{array}$} & \multirow[b]{2}{*}{$\begin{array}{l}P \text { compared to whol } \\
\text { sample }\end{array}$} \\
\hline & $\mathbf{N}$ & $\%$ & $\mathbf{N}$ & $\%$ & & & & \\
\hline Caregiving with FT work & 215 & 5.4 & 227 & 3.9 & & 6.9 & & \\
\hline Working PT but not caregiving & 239 & 6.7 & 1394 & 24.9 & & 7.8 & & \\
\hline Working FT but not caregiving & 1926 & 52.4 & 1332 & 26.2 & & 17.9 & & \\
\hline Co-resident caregiving & & & & & & & & $<0.001$ \\
\hline Not caregiving & 3502 & 89.9 & 4637 & 85.7 & $<0.001$ & 69.7 & 4642 & \\
\hline Non-resident caregiving & 45 & 1.1 & 85 & 1.5 & & 20.5 & & \\
\hline Co-resident caregiving & 387 & 9.0 & 765 & 12.7 & & 9.8 & & \\
\hline \multicolumn{9}{|l|}{ Covariates } \\
\hline \multicolumn{9}{|l|}{ Age } \\
\hline $16-44 \mathrm{yrs}$ & 1440 & 46.1 & 2424 & 50.2 & $<0.001$ & 40.9 & 0 & $<0.001$ \\
\hline $45-64 \mathrm{yrs}$ & 1449 & 33.3 & 2168 & 35.7 & & 32.1 & & \\
\hline $65+\mathrm{yrs}$ & 1045 & 20.7 & 895 & 14.1 & & 27.1 & & \\
\hline \multicolumn{9}{|l|}{ NS-SEC } \\
\hline Management \& professional & 1809 & 42.6 & 1978 & 33.8 & $<0.001$ & 33.0 & 3972 & $<0.001$ \\
\hline Intermediate & 544 & 14.0 & 1213 & 21.6 & & 30.8 & & \\
\hline Routine \& never worked & 1581 & 43.5 & 2296 & 44.6 & & 36.2 & & \\
\hline \multicolumn{9}{|l|}{ Highest qualification } \\
\hline No qualifications & 464 & 11.1 & 714 & 12.6 & $<0.001$ & 19.1 & 1106 & $<0.001$ \\
\hline GCSE/equivalent & 1151 & 29.7 & 1864 & 32.9 & & 32.9 & & \\
\hline A-level & 848 & 22.6 & 906 & 18.2 & & 19.5 & & \\
\hline Degree/other higher & 1471 & 36.7 & 2003 & 36.4 & & 28.6 & & \\
\hline \multicolumn{9}{|l|}{ Net household income quintiles } \\
\hline Lowest & 411 & 10.4 & 672 & 12.8 & $<0.001$ & 21.9 & 1076 & $<0.001$ \\
\hline 2 & 687 & 17.9 & 1012 & 18.9 & & 21.7 & & \\
\hline 3 & 820 & 21.7 & 1191 & 22.1 & & 19.3 & & \\
\hline 4 & 976 & 24.2 & 1319 & 23.5 & & 18.8 & & \\
\hline Highest & 1040 & 25.9 & 1293 & 22.7 & & 18.3 & & \\
\hline \multicolumn{9}{|l|}{ Longstanding illness or impairment } \\
\hline No & 2391 & 64.9 & 3477 & 65.2 & 0.769 & 58.7 & 1063 & $<0.001$ \\
\hline Yes & 1543 & 35.1 & 2010 & 34.8 & & 41.3 & & \\
\hline \multicolumn{9}{|l|}{ Partnership status } \\
\hline Single & 333 & 13.1 & 628 & 16.1 & $<0.001$ & 28.1 & 1290 & $<0.001$ \\
\hline Married \& living with partner & 2886 & 66.1 & 3519 & 58.9 & & 38.5 & & \\
\hline Separated/divorced & 247 & 5.5 & 669 & 10.8 & & 15.1 & & \\
\hline Widowed & 55 & 1.2 & 137 & 2.3 & & 12.6 & & \\
\hline Cohabiting & 413 & 14.1 & 534 & 12.0 & & 5.7 & & \\
\hline $\begin{array}{l}\text { Number of dependent children }<18 \mathrm{yrs} \text {, } \\
\text { median (IQR) }\end{array}$ & 0 & 0,0 & 0 & 0,1 & $<0.001$ & 0 & 1284 & $<0.001$ \\
\hline \multicolumn{9}{|l|}{ Smoking status } \\
\hline Never smoker & 1380 & 36.0 & 2487 & 45.5 & $<0.001$ & 42.8 & 5008 & 0.277 \\
\hline Ex-smoker & 1798 & 42.3 & 1939 & 34.1 & & 36.1 & & \\
\hline Current smoker & 756 & 21.7 & 1061 & 20.4 & & 21.1 & & \\
\hline
\end{tabular}

Abbreviations: A-level, Advanced level; BMI, body mass index; FT, full-time work; GCSE, General Certificate of Secondary Education; IQR, interquartile range; NS-SEC, National Statistics Socio Economic Classification; PT, part-time work; SD, standard deviation. Actual numbers and weighted percentages shown. Descriptive statistics shown for those with at least one adiposity measure with complete data on all analysis variables $(\mathrm{n}=9,421)$ 
economically advantaged and younger than those with observed data. However complete cases had higher BMI and percentage body fat. The sample size for analyses with BMI was 9,190 (58.5\% women), 9,318 (58.2\% women) for waist circumference and 8,778 (59.1\% women) for percentage body fat. The descriptive statistics shown in Table 1 are given for those with at least one adiposity measure who had information on caregiving and covariates $(n=9,421)$.

\section{Statistical analyses}

Linear regression was used to test associations between caregiving (binary) at $\mathrm{tl}$ and BMI, waist circumference and percentage body fat at $\mathrm{t} 2$ ('crude association'). A second model adjusted for age group ('age-adjusted'). A final model was run including longstanding illness or disability, educational attainment, social class, partnership status, number of dependent children, household income and smoking status. In addition, age-caregiving interactions were tested throughout using a Wald test. Where interactions were present, estimates were stratified by age group. The same modelling strategy was applied to the analysis of caregiving characteristics, substituting each additional caregiving variable (caregiving hours, combining work and caregiving, number of people cared for and co-resident caregiving) in place of caregiving status. In order to minimise the problem of multiple comparisons, a Bonferroni corrected $\mathrm{p}$ value was applied based on three adiposity outcomes $(0.05 / 3=0.0167)$. Analyses were gender-stratified, as women are more likely to be informal caregivers and to undertake more intensive caregiving responsibilities. [24] Consequently previous research has shown that the relationship between caregiving and health is generally stronger for women.[6] Also, the relationship between social exposures, such as social roles, and adiposity is generally stronger for women compared to men.[30] Survey weights were applied to account for the design, unequal probabilities of selection, differential non-response and potential sampling errors.

\section{Results}

Women more commonly reported being a caregiver (14.3\%) compared to men (10.1\%) in our sample (Table 1). Women also provided more hours of caregiving per week; $14.8 \%$ of women caregivers were caring for $>20$ hours/week, compared to $10.2 \%$ of men, and men were more likely to report caregiving for $<10$ hours/week. In addition, women caregivers were more likely to be combining caregiving and paid work. Women were also more likely than men to be caring for more than one person and to have caregiving responsibilities within the household. Men had higher mean BMIs and waist circumferences compared to women (mean BMI, men: $28.1 \mathrm{~kg} / \mathrm{m}^{2}$, women: $27.8 \mathrm{~kg} / \mathrm{m}^{2}$; mean waist circumference, men: $99.2 \mathrm{~cm}$, women: $88.6 \mathrm{~cm}$ ). However women had higher percentage body fat than men (mean body fat, men: $23.5 \%$, women: $35.9 \%$ ). Men in the sample were more likely to be in managerial or professional occupations and were more likely to have A-level qualifications, although men and women were similar in the proportion who had at least a degree qualification. Men and women had similar levels of household income in our sample, however men were more likely to be partnered, and to be a current or ex-smoker.

\section{Caregiving and adiposity for men}

Men who were informal caregivers had higher BMIs (1.07, 95\% Confidence Intervals (CI): $0.33,1.81)$, waist circumference $(3.20 \mathrm{~cm}, 95 \%$ CI: $1.62,4.78)$ but not percentage body fat (1.14, $95 \%$ CI: -0.14, 2.41) in crude models (Table 2). However, once age was included these associations were no longer statistically significant. There was little variation in adiposity in relation to hours of caregiving, the number of people cared for or whether the participant was engaged in any co-resident caregiving. Compared to men who were working full-time but not 
Table 2. Associations between informal caregiving and adiposity for UKHLS men.

\begin{tabular}{|c|c|c|c|c|c|c|c|c|c|}
\hline & \multicolumn{3}{|c|}{ Crude association } & \multicolumn{3}{|l|}{ Age-adjusted } & \multicolumn{2}{|c|}{ Fully-adjusted $^{\mathbf{a}}$} & \multirow[b]{2}{*}{ Body fat } \\
\hline & BMI & Waist & Body fat & BMI & Waist & Body fat & BMI & Waist & \\
\hline & $\begin{array}{l}\begin{array}{l}\text { Regression } \\
\text { coeff } \\
(95 \% \mathrm{CI})\end{array} \\
\end{array}$ & $\begin{array}{l}\text { Regression } \\
\text { coeff } \\
(95 \% \mathrm{CI})\end{array}$ & \begin{tabular}{|l} 
Regression \\
coeff \\
$(95 \% \mathrm{CI})$
\end{tabular} & $\begin{array}{l}\text { Regression } \\
\text { coeff } \\
(\mathbf{9 5 \%} \mathrm{CI})\end{array}$ & $\begin{array}{l}\text { Regression } \\
\text { coeff } \\
(\mathbf{9 5 \%} \mathrm{CI})\end{array}$ & $\begin{array}{l}\text { Regression } \\
\text { coeff } \\
(95 \% \mathrm{CI})\end{array}$ & \begin{tabular}{|l} 
Regression \\
coeff \\
$(95 \% \mathrm{CI})$
\end{tabular} & \begin{tabular}{|l} 
Regression \\
coeff \\
$(95 \% \mathrm{CI})$
\end{tabular} & $\begin{array}{l}\text { Regression } \\
\text { coeff } \\
(95 \% \mathrm{CI})\end{array}$ \\
\hline \multicolumn{10}{|l|}{ Caregiving } \\
\hline No & Ref & Ref & Ref & Ref & Ref & Ref & Ref & Ref & Ref \\
\hline Yes & $\begin{array}{l}1.07 \\
(0.33,1.81)\end{array}$ & \begin{tabular}{|l|}
3.20 \\
$(1.62,4.78)$ \\
\end{tabular} & \begin{tabular}{|l}
1.14 \\
$(-0.14,2.41)$ \\
\end{tabular} & $\begin{array}{l}0.64 \\
(-0.09,1.37)\end{array}$ & $\begin{array}{l}1.21 \\
(-0.31,2.73)\end{array}$ & $\begin{array}{l}0.30 \\
(-1.05,1.65)\end{array}$ & \begin{tabular}{|l}
0.50 \\
$(-0.21,1.21)$
\end{tabular} & \begin{tabular}{|l}
0.86 \\
$(-0.65,2.37)$ \\
\end{tabular} & $\begin{array}{l}0.22 \\
(-1.11,1.54)\end{array}$ \\
\hline $\mathrm{R}^{2}$ & 0.43 & 0.51 & 0.15 & 2.53 & 8.41 & 3.33 & 7.06 & 13.97 & 5.45 \\
\hline \multicolumn{10}{|l|}{ Caregiving hours } \\
\hline Not caregiving & \begin{tabular}{|l}
-1.06 \\
$(-1.88,-0.24)$
\end{tabular} & $\begin{array}{l}-3.17 \\
(-4.89,-1.45)\end{array}$ & \begin{tabular}{|l}
-1.45 \\
$(-2.87,-0.02)$
\end{tabular} & \begin{tabular}{|l}
-0.65 \\
$(-1.46,0.16)$
\end{tabular} & $\begin{array}{l}-1.27 \\
(-2.94,0.40)\end{array}$ & $\begin{array}{l}-0.63 \\
(-2.10,0.84)\end{array}$ & $\begin{array}{l}-0.55 \\
(-1.35,0.24)\end{array}$ & \begin{tabular}{|l}
-1.05 \\
$(-2.73,0.62)$
\end{tabular} & $\begin{array}{l}-0.61 \\
(-2.04,0.82)\end{array}$ \\
\hline 1-9 hours/week & Ref & Ref & Ref & Ref & Ref & Ref & Ref & Ref & Ref \\
\hline 10-19 hours/week & $\begin{array}{l}-0.37 \\
(-2.47,1.73)\end{array}$ & $\begin{array}{l}-2.02 \\
(-6.72,2.68)\end{array}$ & \begin{tabular}{|l}
-1.15 \\
$(-4.96,2.65)$ \\
\end{tabular} & \begin{tabular}{|l}
-0.36 \\
$(-2.34,1.61)$ \\
\end{tabular} & \begin{tabular}{|l}
-1.74 \\
$(-5.92,2.43)$
\end{tabular} & $\begin{array}{l}-0.87 \\
(-4.48,2.74) \\
\end{array}$ & \begin{tabular}{|l}
-0.35 \\
$(-2.27,1.58)$
\end{tabular} & \begin{tabular}{|l}
-1.80 \\
$(-5.97,2.37)$ \\
\end{tabular} & $\begin{array}{l}-0.94 \\
(-4.56,2.69)\end{array}$ \\
\hline $20+$ hours/week & $\begin{array}{l}0.53 \\
(-1.86,2.92)\end{array}$ & $\begin{array}{l}2.83 \\
(-1.72,7.38) \\
\end{array}$ & $\begin{array}{l}-2.00 \\
(-6.10,2.10)\end{array}$ & \begin{tabular}{|l}
0.28 \\
$(-2.14,2.69)$ \\
\end{tabular} & \begin{tabular}{|l}
1.53 \\
$(-3.22,6.29)$
\end{tabular} & $\begin{array}{l}-2.59 \\
(-6.86,1.68)\end{array}$ & \begin{tabular}{|l}
-0.16 \\
$(-2.62,2.31)$
\end{tabular} & \begin{tabular}{|l}
0.07 \\
$(-4.60,4.74)$
\end{tabular} & $\begin{array}{l}-3.25 \\
(-7.57,1.07)\end{array}$ \\
\hline $\mathrm{R}^{2}$ & 0.27 & 0.44 & 0.26 & 1.88 & 8.18 & 3.32 & 5.56 & 13.53 & 5.32 \\
\hline \multicolumn{10}{|l|}{$\begin{array}{l}\text { Number of people } \\
\text { cared for }\end{array}$} \\
\hline Not caregiving & $\begin{array}{l}-0.99 \\
(-1.70,- \\
0.29)\end{array}$ & $\begin{array}{l}-3.36 \\
(-5.19,-1.53)\end{array}$ & $\begin{array}{l}-1.13 \\
(-2.44,0.17)\end{array}$ & $\begin{array}{l}-0.55 \\
(-1.24,0.14)\end{array}$ & $\begin{array}{l}-1.37 \\
(-3.16,0.41)\end{array}$ & $\begin{array}{l}-0.32 \\
(-1.63,0.99)\end{array}$ & $\begin{array}{l}-0.43 \\
(-1.11,0.24)\end{array}$ & $\begin{array}{l}-1.08 \\
(-2.85,0.69)\end{array}$ & $\begin{array}{l}-0.26 \\
(-1.58,1.06)\end{array}$ \\
\hline 1 person & Ref & Ref & Ref & Ref & Ref & Ref & Ref & Ref & Ref \\
\hline $2+$ people & $\begin{array}{l}0.24 \\
(-1.53,2.01)\end{array}$ & $\begin{array}{l}-0.52 \\
(-3.43,2.39)\end{array}$ & $\begin{array}{l}0.03 \\
(-3.37,3.42)\end{array}$ & $\begin{array}{l}0.27 \\
(-1.47,2.02)\end{array}$ & $\begin{array}{l}-0.52 \\
(-3.36,2.32)\end{array}$ & $\begin{array}{l}-0.05 \\
(-3.55,3.45)\end{array}$ & $\begin{array}{l}0.22 \\
(-1.54,1.98)\end{array}$ & $\begin{array}{l}-0.70 \\
(-3.71,2.30)\end{array}$ & $\begin{array}{l}-0.14 \\
(-3.61,3.34)\end{array}$ \\
\hline $\mathrm{R}^{2}$ & 0.25 & 0.41 & 0.15 & 1.87 & 8.14 & 3.20 & 5.55 & 13.51 & 5.16 \\
\hline \multicolumn{10}{|l|}{$\begin{array}{l}\text { Combining work } \\
\text { with caregiving }\end{array}$} \\
\hline $\begin{array}{l}\text { Not working or } \\
\text { caregiving }\end{array}$ & $\begin{array}{l}0.32 \\
(-0.15,0.78)\end{array}$ & \begin{tabular}{|l|}
3.60 \\
$(2.32,4.87)$ \\
\end{tabular} & \begin{tabular}{|l|l|}
1.59 \\
$(0.70,2.49)$ \\
\end{tabular} & \begin{tabular}{|l|}
-0.60 \\
$(-1.60,0.40)$ \\
\end{tabular} & \begin{tabular}{|l}
-0.05 \\
$(-0.70,0.60)$
\end{tabular} & $\begin{array}{l}0.54 \\
(-1.21,2.29) \\
\end{array}$ & $\begin{array}{l}0.20 \\
(-0.48,0.88)\end{array}$ & \begin{tabular}{|l}
1.20 \\
$(-0.56,2.96)$ \\
\end{tabular} & $\begin{array}{l}0.05 \\
(-1.30,1.41) \\
\end{array}$ \\
\hline $\begin{array}{l}\text { Caregiving but } \\
\text { not working }\end{array}$ & $\begin{array}{l}0.75 \\
(-0.64,2.14)\end{array}$ & $\begin{array}{l}3.79 \\
(1.40,6.19)\end{array}$ & \begin{tabular}{|l}
1.55 \\
$(-0.79,3.89)$ \\
\end{tabular} & $\begin{array}{l}-0.38 \\
(-1.94,1.18)\end{array}$ & \begin{tabular}{|l}
0.17 \\
$(-1.22,1.55)$
\end{tabular} & \begin{tabular}{|l}
-0.06 \\
$-2.73,2.61)$ \\
\end{tabular} & $\begin{array}{l}0.09 \\
(-1.26,1.44)\end{array}$ & $\begin{array}{l}-0.33 \\
(-2.97,2.31)\end{array}$ & $\begin{array}{l}-0.57 \\
(-3.31,2.17)\end{array}$ \\
\hline $\begin{array}{l}\text { Caregiving with } \\
\text { PT work }\end{array}$ & $\begin{array}{l}2.88 \\
(0.85,4.91)\end{array}$ & $\begin{array}{l}9.05 \\
(3.70,14.40)\end{array}$ & \begin{tabular}{|l}
3.28 \\
$(-2.21,8.77)$
\end{tabular} & $\begin{array}{l}1.83 \\
(-0.30,3.97)\end{array}$ & $\begin{array}{l}2.38 \\
(0.39,4.37)\end{array}$ & $\begin{array}{l}6.22 \\
(1.41,1.03)\end{array}$ & $\begin{array}{l}2.42 \\
(0.64,4.21)\end{array}$ & \begin{tabular}{|l}
6.33 \\
$(2.23,10.44)$
\end{tabular} & $\begin{array}{l}1.95 \\
(-3.11,7.01)\end{array}$ \\
\hline $\begin{array}{l}\text { Caregiving with } \\
\text { FT work }\end{array}$ & $\begin{array}{l}1.11(0.25 \\
1.97)\end{array}$ & $\begin{array}{l}3.93(1.90, \\
5.97)\end{array}$ & $\begin{array}{l}1.44(0.03, \\
2.91)\end{array}$ & \begin{tabular}{|l}
$-1.54(-2.65$ \\
$-0.44)$ \\
\end{tabular} & $\begin{array}{l}0.54(-0.29, \\
1.38)\end{array}$ & $\begin{array}{l}1.36(-0.58 \\
3.30)\end{array}$ & $\begin{array}{l}0.58(-0.26 \\
1.42)\end{array}$ & $\begin{array}{l}1.50(-0.46 \\
3.46)\end{array}$ & $\begin{array}{l}0.51(-0.98, \\
1.99)\end{array}$ \\
\hline $\begin{array}{l}\text { Working PT but } \\
\text { not caregiving }\end{array}$ & $\begin{array}{l}-0.89(-1.67 \\
-0.11)\end{array}$ & $\begin{array}{l}-2.03(-4.35 \\
0.29)\end{array}$ & $\begin{array}{l}-0.55 \\
(-2.11,1.00)\end{array}$ & $\begin{array}{l}-0.55(-1.38 \\
0.29)\end{array}$ & \begin{tabular}{|l}
$-1.00(-1.78$ \\
$-0.22)$
\end{tabular} & $\begin{array}{l}-2.94(-5.14, \\
-0.75)\end{array}$ & $\begin{array}{l}-0.58(-1.35, \\
0.18)\end{array}$ & $\begin{array}{l}-1.62(3.70 \\
0.47)\end{array}$ & $\begin{array}{l}-0.53(-2.03, \\
0.97)\end{array}$ \\
\hline $\begin{array}{l}\text { Working FT but } \\
\text { not caregiving }\end{array}$ & Ref & Ref & Ref & Ref & Ref & Ref & Ref & Ref & Ref \\
\hline $\mathrm{R}^{2}$ & 0.89 & 2.40 & 0.79 & 2.91 & 8.91 & 3.44 & 7.34 & 14.39 & 5.53 \\
\hline \multicolumn{10}{|l|}{$\begin{array}{l}\text { Co-resident } \\
\text { caregiving }\end{array}$} \\
\hline Not caregiving & $\begin{array}{l}-0.94(-1.71, \\
-0.17) \\
\end{array}$ & $\begin{array}{l}-2.70(-4.38, \\
-1.03)\end{array}$ & $\begin{array}{l}-0.98(-2.17 \\
0.22)\end{array}$ & $\begin{array}{l}-0.51(-1.27 \\
0.25)\end{array}$ & $\begin{array}{l}-0.75(-2.33 \\
0.82)\end{array}$ & $\begin{array}{l}-0.16(-1.37 \\
1.04)\end{array}$ & $\begin{array}{l}-0.40(-1.12, \\
0.32)\end{array}$ & $\begin{array}{l}-0.50(-2.04, \\
1.04)\end{array}$ & $\begin{array}{l}-0.14(-1.35, \\
1.06)\end{array}$ \\
\hline $\begin{array}{l}\text { Non-resident } \\
\text { caregiving }\end{array}$ & Ref & Ref & Ref & Ref & Ref & Ref & Ref & Ref & Ref \\
\hline $\begin{array}{l}\text { Co-resident } \\
\text { caregiving }\end{array}$ & $\begin{array}{l}1.15(-1.43, \\
3.74)\end{array}$ & $\begin{array}{l}4.56(-0.25, \\
9.37)\end{array}$ & $\begin{array}{l}1.51(-4.77 \\
7.78)\end{array}$ & $\begin{array}{l}1.15(-1.45 \\
3.75)\end{array}$ & $\begin{array}{l}4.16(-0.80, \\
9.12)\end{array}$ & $\begin{array}{l}1.27(-5.58 \\
8.13)\end{array}$ & $\begin{array}{l}0.91(-1.99 \\
3.81)\end{array}$ & $\begin{array}{l}3.30(-2.16 \\
8.75)\end{array}$ & $\begin{array}{l}0.66(-5.99 \\
7.31)\end{array}$ \\
\hline
\end{tabular}


Table 2. (Continued)

\begin{tabular}{|c|c|c|c|c|c|c|c|c|c|}
\hline & \multicolumn{3}{|c|}{ Crude association } & \multicolumn{3}{|c|}{ Age-adjusted } & \multicolumn{2}{|c|}{ Fully-adjusted $^{\mathrm{a}}$} & \multirow[b]{2}{*}{ Body fat } \\
\hline & BMI & Waist & Body fat & BMI & Waist & Body fat & BMI & Waist & \\
\hline & $\begin{array}{l}\text { Regression } \\
\text { coeff } \\
(95 \% \mathrm{CI})\end{array}$ & $\begin{array}{l}\text { Regression } \\
\text { coeff } \\
(\mathbf{9 5 \%} \mathrm{CI})\end{array}$ & $\begin{array}{l}\text { Regression } \\
\text { coeff } \\
(\mathbf{9 5 \%} \mathrm{CI})\end{array}$ & $\begin{array}{l}\text { Regression } \\
\text { coeff } \\
(\mathbf{9 5 \%} \mathrm{CI})\end{array}$ & $\begin{array}{l}\text { Regression } \\
\text { coeff } \\
(\mathbf{9 5 \%} \mathrm{CI})\end{array}$ & $\begin{array}{l}\text { Regression } \\
\text { coeff } \\
(\mathbf{9 5 \%} \mathrm{CI})\end{array}$ & $\begin{array}{l}\text { Regression } \\
\text { coeff } \\
(\mathbf{9 5 \%} \mathrm{CI})\end{array}$ & $\begin{array}{l}\text { Regression } \\
\text { coeff } \\
(\mathbf{9 5 \%} \mathrm{CI})\end{array}$ & $\begin{array}{l}\text { Regression } \\
\text { coeff } \\
(\mathbf{9 5 \%} \mathrm{CI})\end{array}$ \\
\hline $\mathrm{R}^{2}$ & 0.48 & 0.63 & 0.16 & 2.59 & 8.50 & 3.34 & 7.09 & 14.0 & 5.45 \\
\hline
\end{tabular}

Sample size for BMI analyses, $\mathrm{n}=3,817$; sample size for waist circumference analyses, $\mathrm{n}=3,891$; sample size for percentage body fat analyses, $\mathrm{n}=3,589$

BMI, body mass index; FT, full-time; PT, part-time

${ }^{a}$ Model includes banded age, longstanding health condition, social class, educational attainment, partnership status, number of dependent children, smoking status, and equivalised household income quintiles. Emboldened figures denote statistically significant associations using Bonferroni-corrected $\mathrm{p}=0.0167$

https://doi.org/10.1371/journal.pone.0200777.t002

caregiving, men who weren't working but were caregiving had higher waist circumference, but this was no longer significant after then inclusion of age in the model. Similarly men combining caregiving with full-time work had lower BMIs but this difference was no longer significant in the final model. However men who were combining caregiving with part-time work had higher BMIs and waist circumferences which remained in the fully-adjusted models (BMI: 2.42, 95\% CI: 0.64, 4.21; waist: 6.33, 95\% CI: 2.23, 10.44).

\section{Caregiving and adiposity for women}

In contrast to men, women who were caregivers had higher adiposity than women who were not caregivers (Table 3, BMI: 1.27, 95\% CI: 0.75, 1.79; waist: 2.92, 95\% CI: 1.82, 4.02; body fat: $1.90,95 \%$ CI: 1.21, 2.58). An age-caregiving interaction was present for women; it was found that caregiving was associated with particularly high BMI, waist and percentage body fat for younger women (aged 16-44)(Table 4).

Women who provided more hours of caregiving ( $>20$ hours per week), had mean BMIs which were almost two points higher $(1.96,95 \%$ CI: $0.31,3.61)$ and waists that were, on

Table 3. Associations between informal caregiving and adiposity for UKHLS women.

\begin{tabular}{|c|c|c|c|c|c|c|c|c|c|}
\hline & \multicolumn{2}{|c|}{ Crude association } & \multirow[b]{2}{*}{ Body fat } & \multicolumn{2}{|l|}{ Age-adjusted } & \multirow[b]{2}{*}{ Body fat } & \multicolumn{2}{|c|}{ Fully-adjusted $^{\mathrm{a}}$} & \multirow[b]{2}{*}{ Body fat } \\
\hline & BMI & Waist & & BMI & Waist & & BMI & Waist & \\
\hline & $\begin{array}{l}\text { Regression } \\
\text { coeff } \\
(95 \% \text { CI })\end{array}$ & $\begin{array}{l}\text { Regression } \\
\text { coeff } \\
(95 \% \text { CI })\end{array}$ & $\begin{array}{l}\text { Regression } \\
\text { coeff } \\
(95 \% \mathrm{CI})\end{array}$ & $\begin{array}{l}\text { Regression } \\
\text { coeff } \\
(95 \% \text { CI })\end{array}$ & $\begin{array}{l}\text { Regression } \\
\text { coeff } \\
(95 \% \mathrm{CI})\end{array}$ & $\begin{array}{l}\text { Regression } \\
\text { coeff } \\
(95 \% \text { CI })\end{array}$ & $\begin{array}{l}\text { Regression } \\
\text { coeff } \\
(95 \% \mathrm{CI})\end{array}$ & $\begin{array}{l}\text { Regression } \\
\text { coeff } \\
(95 \% \mathrm{CI})\end{array}$ & $\begin{array}{l}\text { Regression coeff } \\
(95 \% \mathrm{CI})\end{array}$ \\
\hline \multicolumn{10}{|l|}{$\begin{array}{l}\text { Providing informal } \\
\text { care }\end{array}$} \\
\hline No & Ref & Ref & Ref & b & b & b & b & b & b \\
\hline Yes & $\begin{array}{l}1.27 \\
(0.75,1.79)\end{array}$ & $\begin{array}{l}2.92 \\
(1.82,4.02)\end{array}$ & $\begin{array}{l}1.90 \\
(1.21,2.58)\end{array}$ & & & & & & \\
\hline $\mathrm{R}^{2}$ & 0.55 & 0.53 & 0.66 & & & & & & \\
\hline \multicolumn{10}{|l|}{ Caregiving hours } \\
\hline Not caregiving & \begin{tabular}{|l}
-0.84 \\
$(-1.38,-0.29)$
\end{tabular} & $\begin{array}{l}-2.16 \\
(-3.43,-0.89)\end{array}$ & $\begin{array}{l}-1.62 \\
(-2.39,-0.85)\end{array}$ & $\begin{array}{l}-0.55 \\
(-1.10,-0.01)\end{array}$ & $\begin{array}{l}-1.23 \\
(-2.50,0.05)\end{array}$ & $\begin{array}{l}-0.95 \\
(-1.72,-0.17)\end{array}$ & $\begin{array}{l}-0.51 \\
(-1.04,0.03)\end{array}$ & $\begin{array}{l}-1.12 \\
(-2.38,0.15)\end{array}$ & $\begin{array}{l}-0.89 \\
(-1.66,-0.11)\end{array}$ \\
\hline 1-9 hours/week & Ref & Ref & Ref & Ref & Ref & Ref & Ref & Ref & Ref \\
\hline $\begin{array}{l}\begin{array}{l}10-19 \text { hours/ } \\
\text { week }\end{array} \\
\end{array}$ & \begin{tabular}{|l}
0.81 \\
$(-0.63,2.25)$ \\
\end{tabular} & $\begin{array}{l}0.62 \\
(-1.92,3.16)\end{array}$ & \begin{tabular}{|l}
0.36 \\
$(-1.20,1.91)$
\end{tabular} & \begin{tabular}{|l}
0.79 \\
$(-0.65,2.23)$
\end{tabular} & $\begin{array}{l}0.41 \\
(-2.15,2.98)\end{array}$ & $\begin{array}{l}0.25(-1.37 \\
1.87)\end{array}$ & $\begin{array}{l}0.62 \\
(-0.84,2.09)\end{array}$ & $\begin{array}{l}-0.07(-2.65, \\
2.51)\end{array}$ & $\begin{array}{l}-0.01(-1.62, \\
1.61)\end{array}$ \\
\hline $20+$ hours/week & $\begin{array}{l}1.96 \\
(0.31,3.61)\end{array}$ & $\begin{array}{l}4.39 \\
(1.06,7.72)\end{array}$ & $\begin{array}{l}1.48 \\
(-0.30,3.26)\end{array}$ & $\begin{array}{l}1.81 \\
(0.18,3.44)\end{array}$ & $\begin{array}{l}3.83 \\
(0.57,7.08)\end{array}$ & $\begin{array}{l}1.21(-0.55 \\
2.96)\end{array}$ & $\begin{array}{l}1.29 \\
(-0.29,2.88)\end{array}$ & $\begin{array}{l}2.54(-0.59 \\
5.68)\end{array}$ & $\begin{array}{l}0.55(-1.12 \\
2.23)\end{array}$ \\
\hline
\end{tabular}


Table 3. (Continued)

\begin{tabular}{|c|c|c|c|c|c|c|c|c|c|}
\hline & \multicolumn{2}{|c|}{ Crude association } & \multirow[b]{2}{*}{ Body fat } & \multicolumn{2}{|l|}{ Age-adjusted } & \multirow[b]{2}{*}{ Body fat } & \multicolumn{2}{|c|}{ Fully-adjusted $^{\mathrm{a}}$} & \multirow[b]{2}{*}{ Body fat } \\
\hline & BMI & Waist & & BMI & Waist & & BMI & Waist & \\
\hline & $\begin{array}{l}\text { Regression } \\
\text { coeff } \\
(\mathbf{9 5 \%} \mathrm{CI})\end{array}$ & $\begin{array}{l}\text { Regression } \\
\text { coeff } \\
(\mathbf{9 5 \%} \mathrm{CI})\end{array}$ & $\begin{array}{l}\text { Regression } \\
\text { coeff } \\
(\mathbf{9 5 \%} \mathrm{CI})\end{array}$ & $\begin{array}{l}\text { Regression } \\
\text { coeff } \\
(\mathbf{9 5 \%} \mathrm{CI})\end{array}$ & $\begin{array}{l}\text { Regression } \\
\text { coeff } \\
(95 \% \mathrm{CI})\end{array}$ & $\begin{array}{l}\text { Regression } \\
\text { coeff } \\
(\mathbf{9 5 \%} \text { CI })\end{array}$ & $\begin{array}{l}\text { Regression } \\
\text { coeff } \\
(\mathbf{9 5 \%} \mathrm{CI})\end{array}$ & $\begin{array}{l}\text { Regression } \\
\text { coeff } \\
(\mathbf{9 5 \%} \mathrm{CI})\end{array}$ & $\begin{array}{l}\text { Regression coeff } \\
(95 \% \mathrm{CI})\end{array}$ \\
\hline $\mathrm{R}^{2}$ & 0.75 & 0.71 & 0.72 & 2.64 & 5.43 & 6.24 & 6.70 & 10.85 & 9.04 \\
\hline \multicolumn{10}{|l|}{$\begin{array}{l}\text { Number of people } \\
\text { cared for }\end{array}$} \\
\hline Not caregiving & $\begin{array}{l}-0.74 \\
(-1.24,-0.23)\end{array}$ & $\begin{array}{l}-1.94 \\
(-3.11,-0.76)\end{array}$ & $\begin{array}{l}-1.49 \\
(-2.24,-0.73)\end{array}$ & \begin{tabular}{|l}
-0.48 \\
$(-1.00,0.04)$
\end{tabular} & $\begin{array}{l}-1.08(-2.27 \\
0.11)\end{array}$ & $\begin{array}{l}-0.87(-1.64 \\
-0.10)\end{array}$ & $\begin{array}{l}-0.38(-0.90 \\
0.14)\end{array}$ & $\begin{array}{l}-0.88(-2.07 \\
0.32)\end{array}$ & $\begin{array}{l}-0.74(-1.50 \\
0.03)\end{array}$ \\
\hline 1 person & Ref & Ref & Ref & Ref & Ref & Ref & Ref & Ref & Ref \\
\hline $2+$ people & $\begin{array}{l}1.71 \\
(0.52,2.89)\end{array}$ & $\begin{array}{l}3.14 \\
(0.73,5.55)\end{array}$ & $\begin{array}{l}1.34 \\
(0.01,2.66)\end{array}$ & $\begin{array}{l}1.54 \\
(0.35,2.73)\end{array}$ & $\begin{array}{l}2.54(0.08 \\
4.99)\end{array}$ & $\begin{array}{l}0.98(-0.42, \\
2.37)\end{array}$ & $\begin{array}{l}1.35(0.18 \\
2.53)\end{array}$ & $\begin{array}{l}1.95(-0.43, \\
4.33)\end{array}$ & $\begin{array}{l}0.76(-0.62 \\
2.13)\end{array}$ \\
\hline $\mathrm{R}^{2}$ & 0.80 & 0.69 & 0.74 & 2.67 & 5.77 & 6.25 & 7.00 & 10.84 & 9.69 \\
\hline \multicolumn{10}{|l|}{$\begin{array}{l}\text { Combining work } \\
\text { with caregiving }\end{array}$} \\
\hline $\begin{array}{l}\text { Not working or } \\
\text { caregiving }\end{array}$ & $\begin{array}{l}0.28-0.27 \\
0.84)\end{array}$ & $\begin{array}{l}2.39(1.16 \\
3.63)\end{array}$ & $\begin{array}{l}1.04(0.21, \\
1.88)\end{array}$ & b & b & $\mathrm{b}$ & $\mathrm{b}$ & $\mathrm{b}$ & $\mathrm{b}$ \\
\hline $\begin{array}{l}\text { Caregiving but } \\
\text { not working }\end{array}$ & $\begin{array}{l}1.43(0.55 \\
2.31)\end{array}$ & $\begin{array}{l}4.97(3.12, \\
6.82)\end{array}$ & $\begin{array}{l}2.78(1.66 \\
3.90)\end{array}$ & & & & & & \\
\hline $\begin{array}{l}\text { Caregiving with } \\
\text { PT work }\end{array}$ & $\begin{array}{l}0.94(-0.07 \\
1.96)\end{array}$ & $\begin{array}{l}2.27(0.22 \\
4.32)\end{array}$ & $\begin{array}{l}1.77(0.58 \\
2.95)\end{array}$ & & & & & & \\
\hline $\begin{array}{l}\text { Caregiving with } \\
\text { FT work }\end{array}$ & $\begin{array}{l}1.36(0.40, \\
2.31)\end{array}$ & $\begin{array}{l}3.64(1.49, \\
5.79)\end{array}$ & $\begin{array}{l}2.40(1.08 \\
3.73)\end{array}$ & & & & & & \\
\hline $\begin{array}{l}\text { Working PT but } \\
\text { not caregiving }\end{array}$ & $\begin{array}{l}-0.44(-0.98 \\
0.10)\end{array}$ & $\begin{array}{l}-0.45(-1.71 \\
0.81)\end{array}$ & $\begin{array}{l}0.15(-0.68 \\
0.99)\end{array}$ & & & & & & \\
\hline $\begin{array}{l}\text { Working FT but } \\
\text { not caregiving }\end{array}$ & Ref & Ref & Ref & & & & & & \\
\hline $\mathrm{R}^{2}$ & 0.78 & 1.36 & 0.93 & & & & & & \\
\hline \multicolumn{10}{|l|}{$\begin{array}{l}\text { Co-resident } \\
\text { caregiving }\end{array}$} \\
\hline Not caregiving & $\begin{array}{l}-1.11(-1.63, \\
-0.59)\end{array}$ & $\begin{array}{l}-2.59(-3.69, \\
-1.48)\end{array}$ & $\begin{array}{l}-1.78(-2.47 \\
-1.09) \\
\end{array}$ & \begin{tabular}{|l}
$-0.81(-1.34$, \\
$-0.28)$
\end{tabular} & $\begin{array}{l}-1.60(-2.72, \\
-0.48)\end{array}$ & $\begin{array}{l}-1.07(-1.77 \\
-0.37)\end{array}$ & \begin{tabular}{|l}
$-0.71(-1.24$ \\
$-0.18)$
\end{tabular} & \begin{tabular}{|l}
$-1.39(-2.52$, \\
$-0.26)$
\end{tabular} & $\begin{array}{l}-0.93(-1.63 \\
-0.23) \\
\end{array}$ \\
\hline $\begin{array}{l}\text { Non-resident } \\
\text { caregiving }\end{array}$ & Ref & Ref & Ref & Ref & Ref & Ref & Ref & Ref & Ref \\
\hline $\begin{array}{l}\text { Co-resident } \\
\text { caregiving }\end{array}$ & $\begin{array}{l}-1.49(-0.53, \\
3.50)\end{array}$ & $\begin{array}{l}3.08(-0.86, \\
7.01)\end{array}$ & $\begin{array}{l}1.08(-1.07 \\
3.23)\end{array}$ & $\begin{array}{l}1.35(-0.61, \\
3.31)\end{array}$ & $\begin{array}{l}2.47(-1.42 \\
6.35)\end{array}$ & $\begin{array}{l}0.89(-1.28 \\
3.06)\end{array}$ & $\begin{array}{l}0.82(-1.08 \\
2.73)\end{array}$ & $\begin{array}{l}0.86(-2.86 \\
4.58)\end{array}$ & $\begin{array}{l}0.31(-1.78, \\
2.39)\end{array}$ \\
\hline $\mathrm{R}^{2}$ & 0.64 & & 0.68 & 2.54 & 5.71 & 6.22 & 6.87 & 10.79 & 9.67 \\
\hline
\end{tabular}

Sample size for BMI analyses, $\mathrm{n}=5373$; sample size for waist circumference analyses, $\mathrm{n}=5427$; sample size for percentage body fat analyses, $\mathrm{n}=5189 \mathrm{BMI}-\mathrm{body}$ mass index; FT-full-time; PT-part-time

${ }^{a}$ Model includes banded age, longstanding health condition, social class, educational attainment, partnership status, number of dependent children, smoking status, and equivalised household income quintiles. Emboldened figures denote statistically significant associations using Bonferroni-corrected $\mathrm{p}=0.0167$

${ }^{\mathrm{b}}$ Age- and fully-adjusted models not shown as age interactions were statistically significant. Age-stratified associations shown in Table 4.

average, more than four centimetres larger $(4.39 \mathrm{~cm}, 95 \%$ CI: $1.06 \mathrm{~cm}, 7.72 \mathrm{~cm})$. However upon inclusion of age, these associations were no longer statistically significant according to the Bonferroni corrected p-value. Also, women who were caring for at least two people had higher adiposity but these associations no longer remained after inclusion of all covariates. No association between residential or non-residential caregiving and adiposity was observed.

Compared to women who were working full-time and not caregiving, women who were working full-time combined with caregiving had higher levels of adiposity as indicated by all 
Table 4. Associations between informal caregiving, combining caregiving and work with adiposity for UKHLS women stratified by age group.

\begin{tabular}{|c|c|c|c|c|c|c|}
\hline \multirow[t]{2}{*}{ BMI } & \multicolumn{3}{|l|}{ Crude association } & \multicolumn{3}{|l|}{ Fully-adjusted $^{\mathrm{a}}$} \\
\hline & $16-44 \mathrm{yrs}$ & 45-64 yrs & $65+\mathrm{yrs}$ & $16-44 \mathrm{yrs}$ & 45-64 yrs & $65+y r s$ \\
\hline \multicolumn{7}{|l|}{ Providing informal care } \\
\hline No & Ref & Ref & Ref & Ref & Ref & Ref \\
\hline Yes & $1.94(1.04,2.85)$ & $0.63(-0.10,1.35)$ & $-0.42(-1.57,0.72)$ & $1.42(0.49,2.35)$ & $0.60(-0.12,1.32)$ & $-0.08(-1.31,1.16)$ \\
\hline $\mathrm{R}^{2}$ & 0.88 & 0.18 & 0.08 & 5.46 & 6.23 & 8.80 \\
\hline \multicolumn{7}{|l|}{ Combining work with caregiving } \\
\hline Not working or caregiving & $-0.23(-1.14,0.67)$ & $-0.05(-0.95,0.86)$ & $1.69(-1.55,4.91)$ & $-1.34(-2.24,-0.44)$ & $-1.30(-2.28,-0.32)$ & $-0.31(-3.57,2.95)$ \\
\hline Caregiving but not working & $2.21(0.67,3.75)$ & $0.53(-0.94,1.99)$ & $1.20(-2.32,4.73)$ & $0.69(-0.87,2.25)$ & $-0.59(-2.03,0.85)$ & $-0.46(-3.99,3.07)$ \\
\hline Caregiving with PT work & $1.07(-0.37,2.51)$ & $0.08(-1.12,1.29)$ & $0.79(-2.84,4.41)$ & $-0.69(-2.23,0.85)$ & $-0.49(-1.64,0.66)$ & $-0.22(-3.74,3.29)$ \\
\hline Caregiving with FT work & $2.37(0.49,4.26)$ & $-0.13(-1.37,1.10)$ & $7.28(4.06,10.51)$ & $2.07(0.16,3.99)$ & $-0.17(-1.38,1.03)$ & $7.06(3.38,10.74)$ \\
\hline Working PT but not caregiving & $-0.08(-0.84,0.68)$ & $-1.29(-2.15,-0.43)$ & $1.52(-1.68,4.71)$ & $-1.04(-1.90,-0.18)$ & $-1.88(-2.74,-1.03)$ & $0.02(-3.53,3.57)$ \\
\hline Working FT but not caregiving & Ref & Ref & Ref & Ref & Ref & Ref \\
\hline $\mathrm{R}^{2}$ & 0.99 & 1.03 & 0.44 & 6.33 & 7.49 & 9.12 \\
\hline \multirow[t]{2}{*}{ Waist } & \multicolumn{3}{|l|}{ Crude association } & \multicolumn{3}{|l|}{ Fully-adjusted $^{\mathbf{a}}$} \\
\hline & $16-44 \mathrm{yrs}$ & 45-64 yrs & $65+\mathrm{yrs}$ & 16-44 yrs & 45-64 yrs & $65+\mathrm{yrs}$ \\
\hline \multicolumn{7}{|l|}{ Providing informal care } \\
\hline No & Ref & Ref & Ref & Ref & Ref & Ref \\
\hline Yes & $4.29(2.31,6.27)$ & $1.35(-0.28,2.98)$ & $-2.53(-5.58,0.51)$ & $2.93(0.86,5.00)$ & $1.28(-0.35,2.90)$ & $-2.21(-5.45,1.04)$ \\
\hline $\mathrm{R}^{2}$ & 0.83 & 0.16 & 0.38 & 7.19 & 6.36 & 8.52 \\
\hline \multicolumn{7}{|l|}{ Combining work with caregiving } \\
\hline Not working or caregiving & $0.64(-1.34,2.62)$ & $0.13(-1.95,2.21)$ & $3.97(-6.24,14.19)$ & $-1.88(-3.80,0.04)$ & $-2.43(-4.62,-0.23)$ & $0.25(-9.76,10.25)$ \\
\hline Caregiving but not working & $6.78(3.70,9.87)$ & $2.34(-1.045 .71)$ & $0.71(-9.75,11.18)$ & $3.23(0.03,6.44)$ & $-0.03(-3.40,3.33)$ & $-2.81(-13.19,7.57)$ \\
\hline Caregiving with PT work & $2.28(-1.18,5.74)$ & $-0.62(-3.31,2.08)$ & $3.76(-6.74,14.26)$ & $-1.99(-5.65,1.67)$ & $-1.68(-4.25,0.88)$ & $2.42(-8.51,13.34)$ \\
\hline Caregiving with FT work & $4.68(0.98,8.38)$ & $-0.14(-3.00,2.72)$ & $34.27(24.17,44.38)$ & $4.01(0.21,7.80)$ & $-0.28(-3.04,2.47)$ & $34.09(22.82,45.36)$ \\
\hline Working PT but not caregiving & $0.25(-1.44,1.94)$ & $-2.53(-4.49,-0.57)$ & $3.16(-8.62,14.94)$ & $-2.09(-3.99,-0.18)$ & $-3.68(-5.65,-1.71)$ & $0.48(-10.88,11.85)$ \\
\hline Working FT but not caregiving & Ref & Ref & Ref & Ref & Ref & Ref \\
\hline $\mathrm{R}^{2}$ & 1.05 & 0.98 & 1.68 & 7.86 & 7.32 & 9.87 \\
\hline \multirow[t]{2}{*}{ Body fat } & \multicolumn{3}{|l|}{ Crude association } & \multicolumn{3}{|l|}{ Fully-adjusted $^{\mathrm{a}}$} \\
\hline & $16-44 \mathrm{yrs}$ & 45-64 yrs & $65+$ yrs & 16-44 yrs & 45-64 yrs & $65+$ yrs \\
\hline \multicolumn{7}{|l|}{ Providing informal care } \\
\hline No & Ref & Ref & Ref & Ref & Ref & Ref \\
\hline Yes & $3.11(1.80,4.42)$ & $0.31(-0.72,1.33)$ & $-0.74(-2.06,0.59)$ & $2.48(1.16,3.80)$ & $0.25(-0.75,1.25)$ & $-0.71(-2.06,0.63)$ \\
\hline $\mathrm{R}^{2}$ & 1.11 & 0.03 & 0.14 & 6.46 & 5.18 & 3.85 \\
\hline \multicolumn{7}{|l|}{ Combining work with caregiving } \\
\hline Not working or caregiving & $-0.002(-1.35,1.35)$ & $-0.11(-1.23,1.02)$ & $2.80(-4.72,10.32)$ & $-1.51(-2.95,-0.07)$ & $-1.24(-2.43,-0.04)$ & $2.40(-5.35,10.14)$ \\
\hline Caregiving but not working & $3.73(1.56,5.89)$ & $0.37(-1.61,2.36)$ & $2.13(-5.70,9.97)$ & $1.71(-0.55,3.97)$ & $-0.65(-2.59,1.29)$ & $1.71(-6.35,9.77)$ \\
\hline Caregiving with PT work & $2.18(0.04,4.33)$ & $-0.35(-1.95,1.23)$ & $1.30(-6.98,9.59)$ & $-0.03(-2.21,2.16)$ & $-0.70(-2.26,0.85)$ & $1.10(-7.24,9.44)$ \\
\hline Caregiving with FT work & $4.08(1.42,6.74)$ & $-0.49(-2.10,1.12)$ & $4.57(-3.01,12.15)$ & $3.67(1.00,6.35)$ & $-0.62(-2.19,0.95)$ & $6.17(-2.29,14.64)$ \\
\hline Working PT but not caregiving & $0.58(-0.60,1.76)$ & $-1.25(-2.36,-0.14)$ & $3.69(-4.18,11.56)$ & $-0.80(-2.07,0.46)$ & $-1.61(-2.77,-0.44)$ & $3.31(-4.71,11.32)$ \\
\hline Working FT but not caregiving & Ref & Ref & Ref & Ref & Ref & Ref \\
\hline $\mathrm{R}^{2}$ & 1.29 & 0.56 & 0.46 & 7.06 & 5.81 & 4.14 \\
\hline
\end{tabular}

Sample size for BMI analyses, $\mathrm{n}=5$ 707; sample size for waist circumference analyses, $\mathrm{n}=5764$; sample size for percentage body fat analyses, $\mathrm{n}=5508$

${ }^{a}$ Model includes banded age, longstanding health condition, social class, educational attainment, partnership status, number of dependent children, smoking status, and equivalised household income quintiles. Emboldened figures denote statistically significant associations using Bonferroni-corrected $\mathrm{p}=0.0167$

https://doi.org/10.1371/journal.pone.0200777.t004

three outcome measures. A significant age-caregiving and work interaction was found. The age-stratified associations presented in Table 4 suggest that this was found to be driven by 
particularly high levels of adiposity amongst younger (16-44 years) and older (65+ years) women who were combining caregiving with full-time work. For instance, women aged 16-44 years combining full-time work with caregiving had BMIs which were more than two points higher $(2.07,95 \%$ CI: $0.16,3.99)$ and women aged 65 or over had BMIs more than seven points higher $(7.06,95 \%$ CI: 3.38, 10.74). The increased adiposity of younger and older women combining full-time work with caregiving was even more pronounced for waist circumference (16-44 years, waist: $4.01,95 \%$ CI: 0.21, 7.80; 65+ years, waist: 34.09, 95\% CI: 22.82, 45.36). Women aged 16-44 years combining full-time work with caregiving also had higher percentage body fat.

\section{Discussion}

Using UK longitudinal data we found that informal caregiving was associated with higher adiposity for women but not men. We found that the association between informal caregiving and adiposity was driven by younger women aged 16-44 showing particularly strong associations with adiposity. Secondly we found that the combination of paid work and caregiving was important for men and women. Men combining part-time work and caregiving had higher adiposity, as did women who were combining full-time work and caregiving, particularly for younger (aged 16-44) and older (aged 65+) women.

Whilst women might be more likely to adopt a caregiving role [24], their health may be impacted upon more negatively than men's. Indeed previous research showed that women caregivers had poorer health than men caregivers $[8,26,27,31,32]$. The gender differences observed may be because women are more likely to be primary caregivers [33] and therefore may experience greater burden and responsibility for the care recipient. Women are also more likely to adopt more intensive caregiving activities, including more hours and the provision of personal care and assistance with activities of daily living [34], the latter of which we were unable to investigate here. Interestingly, we found no association between caregiving hours and adiposity for men or women. This is in contrast to previous research using the UK Census to investigate associations between caregiving and caregiving intensity with self-rated health [20], where the greater the hours of care provided, the greater the negative impact upon health.

We extend previous work, which has largely focused on psychological health, to adiposity. One previous study investigated the relationship between caregiving and weight gain [12] and found that caregiving was associated with increased BMI and obesity, particularly for men. Whilst, for men, this is inconsistent with our findings, this study was based upon a small sample $(n=167)$ of caregivers to a specific patient group (Alzheimer's disease) which may not be generalisable to a broader population. Other work by Hajek and colleagues $[17,35]$ found that men in the German Aging Study who were informal caregivers had increased BMI. This association was not observed for women. Again the discrepancy in findings might come from differences in the study sample, as the present study is a wider population of adults aged 16+.

We additionally found that younger women caregivers had particularly higher levels of adiposity. This may be due to caregiving outside of the normative life stage, possibly combined with work and other family responsibilities, such as the raising of children. Indeed we found that women caregivers who were combining full-time paid work and caregiving responsibilities had higher adiposity, and this was again particularly the case for younger (aged 16-44) and older (aged 65+) women in our sample. We also found that combining part-time paid work and caregiving was associated with increased adiposity for men. Younger carers may also experience a particularly greater loss in social support, especially as caregiving is a non-normative experience during this life stage. Our study is one of the first to investigate caregiving for adults aged $16+$, rather than restricting our analyses to mid-life onwards. 
Our findings also suggest that it is important to consider caregiving characteristics and the combination with other social roles. For instance, as mentioned above, we found that caregiving status was not associated with adiposity for men, but the combination of part-time working and caregiving was. Informal caring is time consuming and relatively inflexible and therefore places time constraints on paid work [27]. Previous research using the UKHLS found that women caregivers were much more likely to leave part-time (OR $=2.64,95 \% \mathrm{CI}: 1.46,4.79)$ or almost full-time work ( $\mathrm{OR}=4.46,95 \% \mathrm{CI}: 2.53,7.88)$, compared to women who were not caregivers [21]. Time constraints also affect the quantity of leisure time available and the time available for preparing healthy, nutritious meals and undertaking physical exercise. Indeed a German study found that informal caregivers had lower participation in sporting activities compared to non-caregiving peers [35]. Additionally, psychosocial stress can directly affect fat deposition processes, and this might be another mechanism through which informal caregiving might result in higher adiposity [16]. Whilst our initial hypothesis was that informal caregiving is likely to be associated with increased adiposity and weight gain, we also acknowledge that psychosocial stress and any physical exertion required might result in weight loss and a reduction in adiposity for some people. Further exploration of our data showed that informal caregivers were not more likely to be underweight as well, although only 27 men and 61 women in our sample were classified as underweight using the World Health Organisation's classification of obesity [36].

\section{Strengths and limitations}

Our study has a few limitations. Firstly, we used complete case analysis. Those with complete data and missing data (Table 1) differed on most characteristics; our sample was more socially advantaged and less likely to be caregiving. Consequently, if we were able to analyse the full sample we'd include more socially disadvantaged people, including more caregiving and people with higher levels of adiposity. Therefore we expect our findings to be an underestimation of the associations seen, had the full data been utilised. Secondly, we only had adiposity data at one wave and this was early in the UKHLS. We were therefore unable to look at adiposity change, control for adiposity prior to caregiving, or to investigate longer-term associations between caregiving and adiposity. We were also unable to rule out the possibility that adiposity preceded informal caregiving. Thirdly, we were unable to explore caregiving duration, caregiving strain, the relationship between the caregiver and care recipient, the reasons the care recipient required care or the specific caregiving activities undertaken. These aspects of caregiving are likely to be important for health. Further research is therefore needed with a different dataset to explore these aspects of caregiving. Despite these limitations our study also had a number of strengths. We used a large, longitudinal UK dataset which allowed us to control for the caregivers' health status at baseline, which would not be possible using a cross-sectional study. Unlike much previous research, this was a general population sample and included people aged 16 and over. Our findings about the heightened risk of younger women caregivers is therefore an important addition to the evidence base on caregiving and health. Finally, BMI, waist circumference and percentage body fat were measured by trained study nurses.

In summary, we found that informal caregiving was associated with higher adiposity, particularly for younger women. The combination of caregiving and paid work responsibilities also appears to be important for adiposity for both men and women. Further research is required to investigate potential mechanisms, including caregiver burden, loss of social support, depression, financial strain, and risky health behaviours. Given the increasing prevalence of informal caregiving and its societal and financial importance, caregiver health should be a public health priority. 


\section{Acknowledgments}

Understanding Society is an initiative funded by the Economic and Social Research Council and various Government Departments, with scientific leadership by the Institute for Social and Economic Research, University of Essex, and survey delivery by NatCen Social Research and Kantar Public. The research data are distributed by the UK Data Service.

\section{Author Contributions}

Conceptualization: Rebecca E. Lacey, Anne McMunn, Elizabeth Webb.

Formal analysis: Rebecca E. Lacey.

Funding acquisition: Rebecca E. Lacey, Anne McMunn, Elizabeth Webb.

Investigation: Rebecca E. Lacey.

Methodology: Rebecca E. Lacey, Anne McMunn, Elizabeth Webb.

Project administration: Elizabeth Webb.

Supervision: Anne McMunn, Elizabeth Webb.

Writing - original draft: Rebecca E. Lacey.

Writing - review \& editing: Rebecca E. Lacey, Anne McMunn, Elizabeth Webb.

\section{References}

1. CarersTrust. Key facts about carers and the people they care for | Carers Trust [Internet]. 2017 [cited 8 Mar 2017]. Available: https://carers.org/key-facts-about-carers-and-people-they-care

2. Hounsell C, Jed Johnson W, Seals Carol Levine E, Stein R, Vuckovic N, Donna Wagner I, et al. Caregiving in the U.S.-AARP 2015 Report. 2015; Available: http://www.caregiving.org/wp-content/uploads/ 2015/05/2015_CaregivingintheUS_Final-Report-June-4_WEB.pdf

3. Feinberg L, Choula R. Understanding the Impact of Family Caregiving on Work [Internet]. Washington DC; 2012. Available: http://www.aarp.org/content/dam/aarp/research/public_policy_institute/ltc/2012/ understanding-impact-family-caregiving-work-AARP-ppi-ltc.pdf

4. ONS. Mortality, 2014-based national population projections reference volume-Office for National Statistics [Internet]. 2016. Available: https://www.ons.gov.uk/peoplepopulationandcommunity/ populationandmigration/populationprojections/compendium/nationalpopulationprojections/2014based referencevolumeseriespp2/chapter4mortality2014basednationalpopulationprojectionsreference volume\#future-prosp

5. LGA. Adult social care funding state of the nation 2016 [Internet]. London; 2016. Available: http://www. local.gov.uk/documents/10180/7632544/1+24+ASCF+state+of+the+nation+2016 WEB.pdf/e5943f2d4dbd-41a8-b73e-da0c7209ec12

6. Vitaliano PP, Zhang J, Scanlan JM. Is Caregiving Hazardous to One's Physical Health? A Meta-Analysis. Psychol Bull. American Psychological Association; 2003; 129: 946-972. https://doi.org/10.1037/ 0033-2909.129.6.946 PMID: 14599289

7. Pinquart M, Sörensen S. Correlates of physical health of informal caregivers: a meta-analysis. J Gerontol B Psychol Sci Soc Sci. Oxford University Press; 2007; 62: P126-37. PMID: 17379673

8. Pinquart $M$, Sörensen $S$. Differences between caregivers and noncaregivers in psychological health and physical health: A meta-analysis. Psychol Ageing. 2003; 18: 250-267.

9. Kiecolt-Glaser JK, Dura JR, Speicher CE, Trask OJ, Glaser R. Spousal caregivers of dementia victims: longitudinal changes in immunity and health. Psychosom Med. 1991; 53: 345-362. https://doi.org/10. 1097/00006842-199107000-00001 PMID: 1656478

10. Pearlin LI, Mullan JT, Semple SJ, Skaff MM. Caregiving and the stress process: an overview of concepts and their measures. Gerontologist. Oxford University Press; 1990; 30: 583-94. https://doi.org/10. 1093/GERONT/30.5.583 PMID: 2276631

11. Roepke SK, Mausbach BT, Von Käne R, Ancoli-Israel S, Harmell AL, Dimsdale JE, et al. The moderating role of personal mastery on the relationship between caregiving status and multiple dimensions of 
fatigue. Int J Geriatr Psychiatry. 2009; 24: 1453-1462. https://doi.org/10.1002/gps.2286 PMID: 19548267

12. Vitaliano PE, Russo J, Scanlan JM, Greeno CG. Weight Changes in Caregivers of Alzheimer's Care Recipients: Psychobehavioral Predictors. 1996; 11: 155-163. PMID: 8726381

13. Kim Y, Duberstein PR, Sörensen S, Larson MR. Levels of depressive symptoms in spouses of people with lung cancer: effects of personality, social support, and caregiving burden. Psychosomatics. 2005; 46: 123-30. https://doi.org/10.1176/appi.psy.46.2.123 PMID: 15774950

14. Schulz R, Newsom J, Mittelmark M, Burton L, Hirsch C, Jackson S. Health effects of caregiving: the caregiver health effects study: an ancillary study of the Cardiovascular Health Study. Ann Behav Med. 1997; 19: 110-116. https://doi.org/10.1007/BF02883327 PMID: 9603685

15. Da Roza Davis JM, Cowen PJ. Biochemical stress of caring. Psychol Med. Cambridge University Press; 2001; 31: 1475-1478. PMID: 11722162

16. Wardle J, Chida Y, Gibson EL, Whitaker KL, Steptoe A. Stress and Adiposity: A Meta-Analysis of Longitudinal Studies. Obesity. Blackwell Publishing Ltd; 2011; 19: 771-778. https://doi.org/10.1038/oby. 2010.241 PMID: 20948519

17. Hajek A, König $\mathrm{H}-\mathrm{H}$. The longitudinal association between informal caregiving and body mass index in the second half of life: findings of the German Ageing Survey. Public Health. 2017; 151: 81-86. https:// doi.org/10.1016/j.puhe.2017.06.022 PMID: 28753532

18. Vlachantoni A, Robards J, Falkingham J, Evandrou M. Trajectories of informal care and health. SSMPopul Heal. 2016; 2: 495-501. https://doi.org/10.1016/j.ssmph.2016.05.009 PMID: 29349165

19. Legg L, Weir CJ, Langhorne P, Smith LN, Stott DJ. Is informal caregiving independently associated with poor health? A population-based study. J Epidemiol Community Health. BMJ Publishing Group Ltd; 2013; 67: 95-7. https://doi.org/10.1136/jech-2012-201652 PMID: 22875077

20. O'Reilly $\mathrm{D}$, Connolly $\mathrm{S}$, Rosato $\mathrm{M}$, Patterson $\mathrm{C}$. Is caring associated with an increased risk of mortality? A longitudinal study. Soc Sci Med. 2008; 67: 1282-1290. https://doi.org/10.1016/j.socscimed.2008.06. 025 PMID: 18667262

21. Carr E, Murray ET, Zaninotto $P$, Cadar D, Head J, Stansfeld S, et al. The Association Between Informal Caregiving and Exit From Employment Among Older Workers: Prospective Findings From the UK Household Longitudinal Study. Journals Gerontol Ser B Psychol Sci Soc Sci. Oxford University Press; 2016; gbw156. https://doi.org/10.1093/geronb/gbw156 PMID: 27927746

22. Hassink WHJ, Van den Berg B. Time-bound opportunity costs of informal care: Consequences for access to professional care, caregiver support, and labour supply estimates. Soc Sci Med. 2011; 73 1508-1516. https://doi.org/10.1016/j.socscimed.2011.08.027 PMID: 21982632

23. Glaser K, Evandrou M, Tomassini $C$. The health consequences of multiple roles at older ages in the UK. Health Soc Care Community. 2005; 13: 470-7. Available: http://www.ncbi.nlm.nih.gov/pubmed/ 16048535 https://doi.org/10.1111/j.1365-2524.2005.00574.x PMID: 16048535

24. Arber S, Ginn J. Gender differences in informal caring. Health Soc Care Community. 2007; 3: 19-31.

25. Vlachantoni A, Evandrou M, Falkingham J, Robards J. Informal care, health and mortality. Maturitas. 2013; 74: 114-118. https://doi.org/10.1016/j.maturitas.2012.10.013 PMID: 23218988

26. Pinquart M, Sörensen S. Gender differences in caregiver stressors, social resources, and health: an updated meta-analysis. J Gerontol B Psychol Sci Soc Sci. Oxford University Press; 2006; 61: P33-45. PMID: 16399940

27. Bauer JM, Sousa-Poza A. Impacts of Informal Caregiving on Caregiver Employment, Health, and Family. J Popul Ageing. 2015; Available: http://link.springer.com/10.1007/s12062-015-9116-0

28. Lynn P. Sample design for Understanding Society [Internet]. Colchester; 2009. Available: https://ideas. repec.org/p/ese/ukhlsp/2009-01.html

29. McFall S, Petersen J, Kaminska O, Lynn P. Understanding Society-UK Household Longitudinal Study: Waves 2 and 3 Nurse Health Assessment, 2010-2012, Guide to Nurse Health Assessment [Internet]. Colchester; 2014. Available: file://S:/Pop_Health/EPH_SDAIWebb/Publications/7251_User_Guide_ Health_Assmt_w2_w3.pdf

30. Lacey RE, Sacker A, Bell S, Kumari M, Worts D, McDonough P, et al. Work-family life courses and BMl trajectories in three British birth cohorts. Int J Obes. 2016; https://doi.org/10.1038/ijo.2016.197 PMID: 27811951

31. Raschick $M$, Ingersoll-Dayton $B$. The costs and rewards of caregiving among aging spouses and adult children. Fam Relat. 2004; 53: 317-325. https://doi.org/10.1111/j.0022-2445.2004.0008.x

32. Yee JL, Schulz R. Gender Differences in Psychiatric Morbidity Among Family Caregivers: A Review and Analysis. Gerontologist. 2000; 40: 147. Available: http://0-search.ebscohost.com.library.simmons. edu/login.aspx?direct=true\&db=a9h\&AN=3172395\&site=ehost-live PMID: 10820918 
33. Allen SM. Gender differences in spousal caregiving and unmet need for care. J Gerontol. 1994; 49: S187-S195. https://doi.org/10.1093/geronj/49.4.S187 PMID: 8014402

34. Kramer BJ, Kipnis S. Eldercare and work-role conflict: toward an understanding of gender differences in caregiver burden. Gerontologist. 1995; 35: 340-348. PMID: 7622087

35. Hajek A, Bock J-O, König H-H. Association of informal caregiving with body mass index and frequency of sporting activities: evidence of a population-based study in Germany. BMC Public Health. BioMed Central; 2017; 17: 755. https://doi.org/10.1186/s12889-017-4786-6 PMID: 28962607

36. WHO. Body mass index-BMI [Internet]. World Health Organization; 18 Oct 2017 [cited 18 Oct 2017]. Available: http://www.euro.who.int/en/health-topics/disease-prevention/nutrition/a-healthy-lifestyle/ body-mass-index-bmi 